\title{
Phosphatidylethanolamine positively regulates autophagy and longevity
}

\author{
P Rockenfeller ${ }^{1}$, M Koska ${ }^{1}$, F Pietrocola ${ }^{2}$, N Minois ${ }^{3}$, O Knittelfelder ${ }^{1}$, V Sica ${ }^{2}$, J Franz ${ }^{1}$, D Carmona-Gutierrez ${ }^{1}$, G Kroemer ${ }^{\star 2,4,5,6,7}$ \\ and $\mathrm{F}$ Madeo $^{*, 1,8}$
}

Autophagy is a cellular recycling program that retards ageing by efficiently eliminating damaged and potentially harmful organelles and intracellular protein aggregates. Here, we show that the abundance of phosphatidylethanolamine (PE) positively regulates autophagy. Reduction of intracellular PE levels by knocking out either of the two yeast phosphatidylserine decarboxylases (PSD) accelerated chronological ageing-associated production of reactive oxygen species and death. Conversely, the artificial increase of intracellular PE levels, by provision of its precursor ethanolamine or by overexpression of the PE-generating enzyme Psd1, significantly increased autophagic flux, both in yeast and in mammalian cell culture. Importantly administration of ethanolamine was sufficient to extend the lifespan of yeast (Saccharomyces cerevisiae), mammalian cells (U2OS, H4) and flies (Drosophila melanogaster). We thus postulate that the availability of PE may constitute a bottleneck for functional autophagy and that organismal life or healthspan could be positively influenced by the consumption of ethanolamine-rich food.

Cell Death and Differentiation (2015) 22, 499-508; doi:10.1038/cdd.2014.219; published online 9 January 2015

Phosphatidylethanolamine (PE) is a phospholipid found in all living organisms. Together with phosphatidylcholine (PC), phosphatidylserine (PS) and phosphatidylinositol (PI), PE represents the backbone of most biological membranes. $\mathrm{PE}$ is the second-most abundant phospholipid in mammalian membranes ranging from 20 to $50 \%{ }^{1}$ In yeast, PE is essential for growth and is generated through four different enzymatic pathways: ${ }^{2} \mathrm{PE}$ can be produced by decarboxylation of PS, as a first option at the mitochondrial membrane via phosphatidylserine decarboxylase 1 (Psd1) $)^{3,4}$ or, as a second, option at the Golgi and vacuolar membranes through phosphatidylserine decarboxylase 2 (Psd2). ${ }^{5}$ As a third possibility, PE can be produced from actively retrieved extracellular ethanolamine, ${ }^{6,7}$ which is cytidine 5'-diphosphate-activated $^{8}$ and then coupled to diacylglycerol to generate PE. ${ }^{9}$ The fourth, scarcely employed PE-generating pathway is based on the lysophospholipid acylation of lyso-PE. Importantly, PE does not spontaneously assemble in bilayers and rather incorporates into curved structures, such as the inverted hexagonal phase. ${ }^{10}$ The physiological function of non-bilayer lipids in membranes is considered to reside in their interaction with membrane proteins via the membrane lateral pressure $^{10}$ and membrane tethering and fusion processes, which are relevant for autophagy. ${ }^{11}$

The term 'autophagy' describes a degradation process affecting intracellular components (for a review see, 12, 13) which as an important cytoprotective mechanism, is closely linked to ageing. Autophagy mainly differs from the proteasomal pathway, the other major cellular degradation mechanism, in two aspects. First, autophagy can degrade large particles or whole organelles and second, the final degradation occurs in the lysosome/vacuole and not at the proteasome. Prior to the actual degradation, the cargo is gathered in autophagic particles, which are surrounded by a characteristic doublemembrane. However, the origin of these autophagosomal membranes is still controversial and might actually depend on the mode of autophagy induction. ${ }^{14,15}$ Among the discussed membrane sources are the Golgi apparatus, the endosplamic reticulum (ER) or the mitochondrion-associated membrane, which is formed at the interface between the ER. ${ }^{16}$ In higher eukaryotes autophagic membranes are enriched in PE with a high degree of unsaturation, ${ }^{17}$ similarly to the PE species found in mitochondria. ${ }^{14,18}$ Moreover, the pre-autophagosomal structure or phagophore assembly site (PAS), which appears at the very beginning of autophagosome formation, already harbours Atg9, an autophagy-related transmembrane protein that shuttles between mitochondria and the PAS structure in yeast. ${ }^{19}$

Importantly, PE also functions as an anchor to autophagosomal membranes for the autophagy-related protein Atg8 in yeast $^{20}$ and its mammalian orthologue LC ${ }^{21,22}$ This PE anchor is provided to LC3/Atg8 post-translationally in a process called lipidation. First, LC3/Atg8 is carboxyterminally cleaved by proteases from the Atg4 family. 23,24 Subsequently, the remaining C-terminal glycine is coupled to

\footnotetext{
${ }^{1}$ Institute of Molecular Biosciences, NAWI Graz, University of Graz, Humboldtstr. 50, 8010 Graz, Austria; ${ }^{2}$ INSERM U848, Villejuif, Paris, France; ${ }^{3}$ Biomedical Sciences Research Complex, University of St Andrews, St Andrews, UK; ${ }^{4}$ Metabolomics Platform, Institut Gustave Roussy, Villejuif, Paris, France; ${ }^{5}$ Centre de Recherche des Cordeliers, Paris, France; ${ }^{6}$ Pôle de Biologie; Hôpital Européen Georges Pompidou, AP - HP, Paris, France; ${ }^{7}$ Université Paris Descartes, Sorbonne Paris Cité, Paris, France and ${ }^{8}$ BioTechMed-Graz, Humboldtstr. 50, 8010 Graz, Austria

${ }^{*}$ Corresponding author: G Kroemer or F Madeo, Institute of Molecular Biosciences, Karl-Franzens-University of Graz, 8010 Graz, Austria. Tel: +43 3163801507 ; Fax: +43 316380 8878; E-mail: kroemer@ orange.fr or frank.madeo@uni-graz.at

Abbreviations: DHE, Dihydroethidium; ER, endoplasmic reticulum; PE, phosphatidylethanolamine; PC, phosphatidylcholine; PI, phosphatidylinositol; PS, phosphatidylserine; Psd/Pisd, phosphatidylserine decarboxylase; PAS, pre-autophagosomal structure/phagophore assembly site; ROS, reactive oxygen species; PI, propidium iodide

Received 30.6.14; revised 17.11.14; accepted 19.11.14; Edited by M Piacentini; published online 09.1.15
} 
$P E$ in a series of ubiquitination-like reactions involving diverse Atg-proteins. ${ }^{20,25-27}$ In vitro, Atg8-PE causes hemifusion of vesicles, which argues for its potential role in autophagosomal phagophore expansion. ${ }^{11,28}$ Consistently, semisynthetic LC3$\mathrm{PE}$ has recently been described to stimulate membrane tethering and fusion. ${ }^{29}$ We thus reasoned that the overall abundance of PE might be critical for PE-lipidation of LC3/Atg8 and could thus regulate autophagosomal membrane formation. Therefore, we tested whether increasing cellular PE levels might have an impact on autophagy and lifespan regulation.

Here, we report that knock-out of PSD1 or PSD2 shortens the chronological lifespan of $S$. cerevisiae, whereas PSD1overexpression enhances the autophagic capacity and increases longevity. Furthermore, external administration of ethanolamine increases endogenous PE levels, enhances autophagic flux and extends the lifespan of yeast, mammalian cells in culture and flies (Drosophila melanogaster).

\section{Results}

A genetic screen identifies $p s d 1 \Delta$ and $p s d 2 \Delta$ as progeroid yeast strains. We designed a screen to identify genes involved in phospholipid anabolism and catabolism that might have an impact on ageing. For this purpose, we performed chronological ageing experiments with a subset of yeast strains deleted for non-essential genes known to be involved in phospholipid metabolism. At day 3 of the chronological ageing experiment, we measured the levels of reactive oxygen species (ROS) by assessing the ROS-driven oxidation of non-fluorescent dihydroethidium (DHE) to fluorescent ethidium by cytofluorometry. This screening procedure led to the identification of three genes whose deletion caused an ageing-dependent raise in ROS generation (Figure 1a): isc $1 \Delta$, psd1 $\Delta$ and psd2 $\Delta$. ISC1 encodes an inositol phosphosphingolipid phospholipase, which produces ceramide. Interestingly, deletion of ISC1 has been previously reported to decrease chronological lifespan, ${ }^{30}$ thus validating our screen results. $P S D 1$ and $P S D 2$ encode phosphatidylserine decarboxylases, which similarly convert PS to PE, and are located in distinct cellular organelles (see Introduction). As these two enzymes have not been associated with yeast aging, we decided to focus our study on these enzymes and their products.

We confirmed the premature ageing phenotype of the PSD1 and PSD2 knock outs by clonogenic survival plating (Figure $1 \mathrm{~b}$ ) and assessed the PE- and PC-abundance at day 3 of ageing by HPLC-assisted analyses of lipid extracts (Figure 1c). PSD1 deletion had a more pronounced inhibitor effect on both PE synthesis and yeast ageing. This is consistent with a previous report underscoring that, at least in standard culture conditions, Psd1 is the predominant phosphatidylserine decarboxylase, the abrogation of which disturbs homeostasis of PE and a number of phospholipid species including PS and PI which can be overcome by the administration of ethanolamine..$^{31}$

As previous works have also shown that $P S D 1$ deletion has a strong impact on mitochondrial function, ${ }^{32,33}$ we examined to which extent the reduction in survival upon chronological
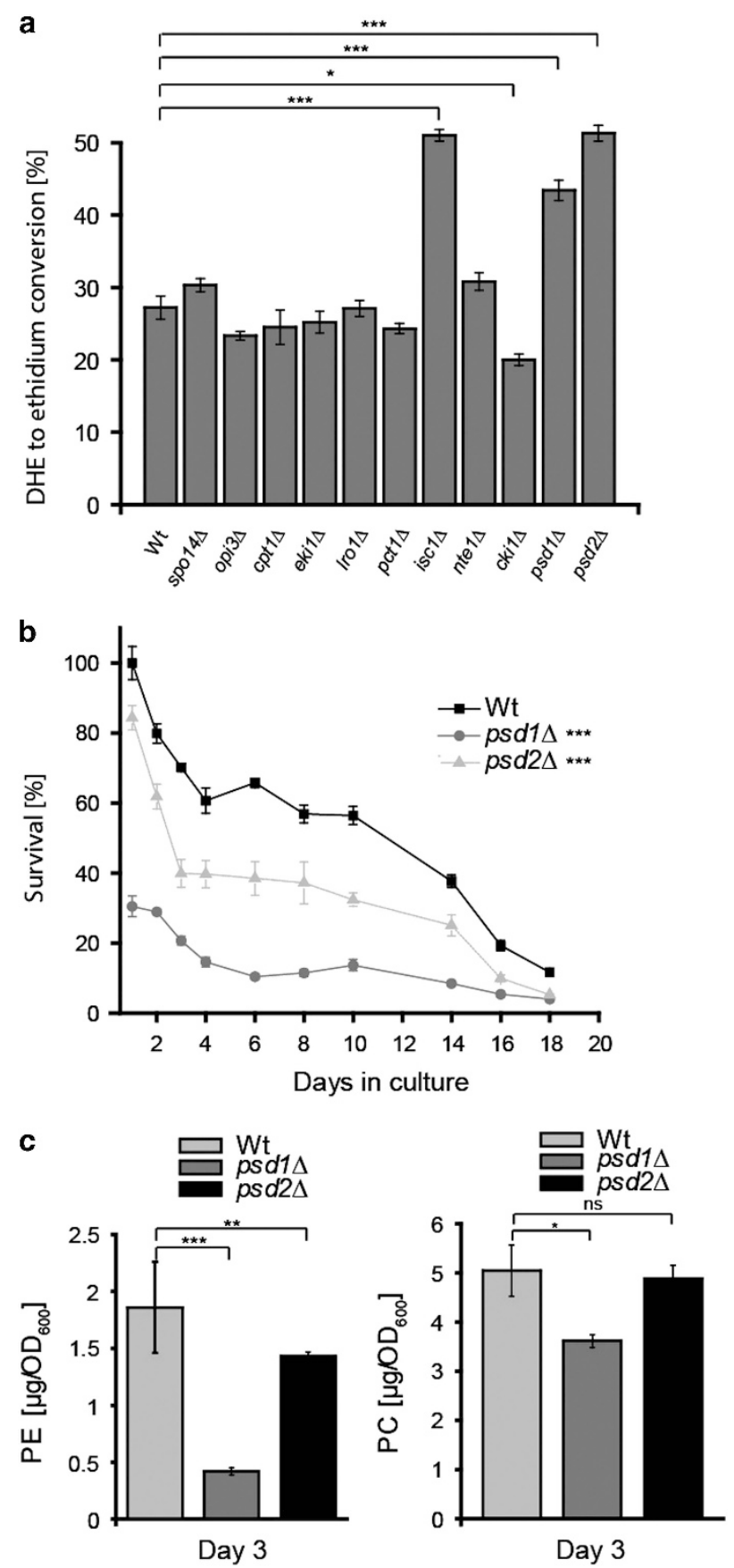

Figure 1 Identification of $p s d 1 \Delta$ and $p s d 2 \Delta$ as progeroid yeast strains. (a) The ROS-driven conversion of DHE to ethidium was measured at day three of chronological ageing in eleven yeast strains deficient for different enzymes involved in phospholipid synthesis. (b) The chronological ageing curve, which is based on the clonogenic survival, is shown for $p s d 1 \Delta$ and psd2 $\Delta$ in comparison with the wild type. (c) Phosphatidylethanolamine (PE) and phosphatidyl choline (PC) were quantified by HPLC-ELSD of lipid extracts from wild-type psd1 $\Delta$ and $p s d 2 \Delta$ cells at day three of chronological ageing

ageing might be a consequence of general mitochondrial dysfunction or specific to the deletion of PSD activity. We thus generated $p s d 1 \Delta$ and $p s d 2 \Delta$ strains, which are additionally rendered defective for respiration owing to loss of mitochondrial DNA $\left(\mathrm{Rho}^{\circ}\right)$. These $\mathrm{Rho}^{\circ}$ strains showed reduced 
survival rates compared with their $\mathrm{Rho}^{+}$counterparts starting from day 2 of chronological ageing (Supplementary Figure $\mathrm{S} 1 \mathrm{~A})$. Still, deletion of mitochondrial DNA exacerbates the premature ageing phenotype of $p s d 1 \Delta$ indicating that mitochondria are at least partially functional in psd1 $\Delta$.

In addition, experiments using growth media containing the non-fermentable carbon source glycerol (Supplementary Figure S1B,C, Supplementary Results) suggest that lack of Psd1 can be compensated over time, presumably by Psd2 activity, providing enough $P E$ to establish functional mitochondria with a sufficient respiratory capacity. We summarise that although during early stages of chronological ageing mitochondrial defects are occurring in $p s d 1 \Delta$, at later time points other effects of PSD1 deletion such as autophagy may become prominent.

PSD1 overexpression extends yeast chronological lifespan and increases autophagy. As the deletion of Psd1 activity decreased yeast lifespan, we wondered whether PSD1 overexpression in wild-type yeast would have the opposite effect. First, we confirmed functionality of the PSD1 overexpression (Figure 2a, Supplementary Results, Supplementary Figure S2A, B). Quantification of total cellular $P E$ levels revealed that $P E$ was significantly higher in cells overexpressing PSD1 (Figure 2b), where PC levels remained unchanged at least on days 1 to 3 (Supplementary Figure S2C). Next we demonstrated that indeed, PSD1 overexpression increased the clonogenic survival (Figure 2c) and decreased ROS accumulation (Figure 2d) during chronological ageing. Interestingly, this increased PEabundance correlated with an upregulation of autophagy, as detected by GFP-Atg8 immuno blotting (Figure $2 e$, Supplementary Figure S2D). The free GFP band, which indicates vacuolar degradation of GFP-Atg8, represents a measure for successful autophagy execution. ${ }^{34,35}$ PSD1 overexpression led to an increase in the intensity of the band corresponding to free GFP at days 2 and 3 of ageing relative to control cells transformed with vector only (Figure $2 \mathrm{e}$ ). In addition, we evaluated microscopically whether vacuoles were GFP-positive, which is indicative for completed autophagy. Indeed, PSD1 overexpression increased the amount of GFP-positive vacuoles at days 2 and 4 (Figure 2f). Measurement of alkaline phosphatase (ALP) activity (Supplementary Figure S2E), which is known to increase with autophagy in a genetically engineered yeast strain, ${ }^{36,37}$ confirmed that PSD1 overexpression increases autophagy flux. Altogether, these data suggest that a rise in intracellular PE mediated by PSD1 overexpression leads to chronological lifespan extension through increased autophagy.

External supply of ethanolamine enhances the endogenous PE pool, induces autophagy and extends yeast lifespan. We next evaluated the hypothesis that external supply of ethanolamine might additionally increase the intracellular PE pool by conversion into PE through the Kennedy pathway. ${ }^{2}$ Indeed, administration of ethanolamine to yeast culture stimulated a substantial augmentation in intracellular PE levels (Figure 3a). Ethanolamine also caused a concentration-dependent increase in yeast cell survival
(Figure 3b), coupled to a decrease in ROS production (Figure 3c) during chronological ageing. Moreover, ethanolamine enhanced autophagic flux as documented by GFP-Atg8 immunoblotting (Figure 3d, Supplementary Figure S3A), microscopic assessment of the subcellular localisation of GFP-Atg8 (Figures $3 e$ and $f$ ) and measurement of ALP activity (Supplementary Figure S3B). It should be noted that the administration of ethanolamine lead to a small, but significant change in the $\mathrm{pH}$ of culture media (Supplementary Figure S3C). However, this change should not be relevant for the observed positive effects on chronological ageing as the $\mathrm{pH}$ decreased, which is known to negatively impact on chronological ageing. ${ }^{38}$ We performed additional control experiments to exclude the possibility that ethanolamine might exert anti-ageing effects as a nitrogen source or owing to its caloric value. However, administration of neither ammonium sulphate (nitrogen control) nor ethanol (calorie control) did mimic the effects of ethanolamine (Supplementary Figure S3D). We also provide evidence that inhibition of ethanolamine driven PE synthesis via the Kennedy pathway by ECT1 $P C T 1 \triangle$ double deletion abolishes ethanolamine-mediated lifespan extension (Supplementary Figure S3E). Ect1 is the cytidyltransferase preferably activating phosphoethanolamine to be further converted into PE, whereas Pct1 is the cytidyltransferase preferably utilising phosphocholine as substrate to synthesise PC. As the two proteins are redundant in their substrate specificity, only the double knock out entirely inhibits $P E$ synthesis by the Kennedy pathway. In addition, inhibition of autophagy by ATG7 deletion abrogated the beneficial effects of ethanolamine treatment on the chronological lifespan (Figure $3 g$ ) or at least partially reduced its positive effects, depending on the genetic strain background (Supplementary Figure S3F). Our findings thus support the notion that intracellular PE levels may affect the lifespan through regulation of autophagy.

Ethanolamine administration induces autophagy and reduces chronological senescence in mammalian cells. Addition of ethanolamine to human osteosarcoma (U2OS) and neuroblastoma $(\mathrm{H} 4)$ cell cultures resulted in increased PE levels (Figure 4a, Supplementary Figure S4A), whereas the endogenous $P C$ levels remained unaffected (Supplementary Figure S4B, C). Furthermore, the autophagic flux, as measured by LC3 lipidation in the absence and presence of the autophagy inhibitor bafilomycin A1 was also increased as a response to ethanolamine treatment (Figure 4b). The bands corresponding to the lipidated fraction of LC3 (LC3II) showed an increased intensity upon ethanolamine treatment as compared with untreated cells or cells exposed to bafilomycin A1 alone. These data were confirmed by fluorescence microscopic detection of autophagic puncta in U2OS cells stably expressing the autophagic marker GFP-LC3 (Figure 4c). The number of GFP-LC3-positive puncta significantly increased in response to ethanolamine, both in the absence and presence of bafilomycin A1. Of note, the level of ethanolamine-mediated autophagy was equivalent to that observed upon nutrient deprivation (namely cultured in Earl's balanced salt solution), which was used as a positive control 
a

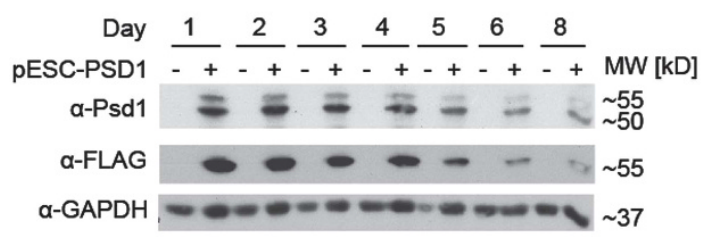

C

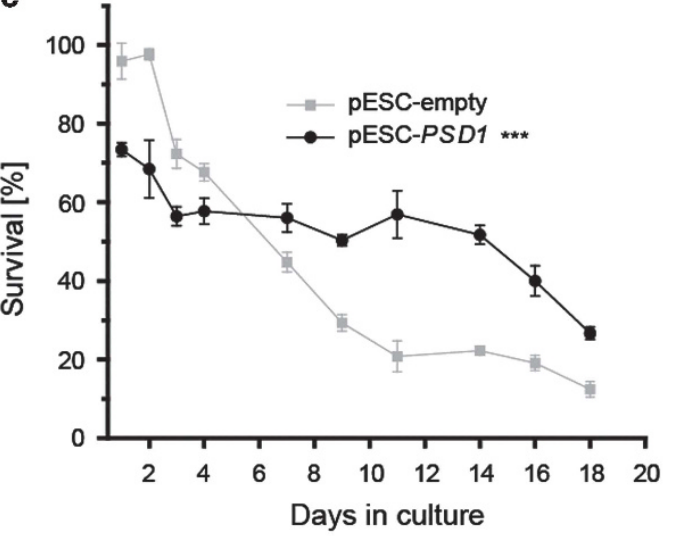

b

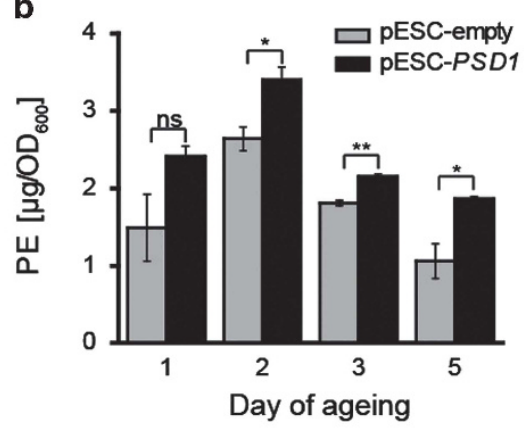

d

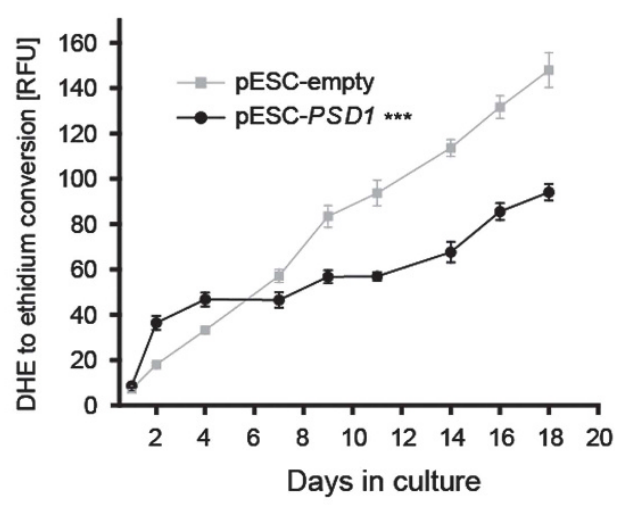

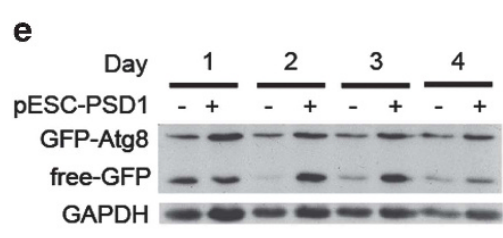

f
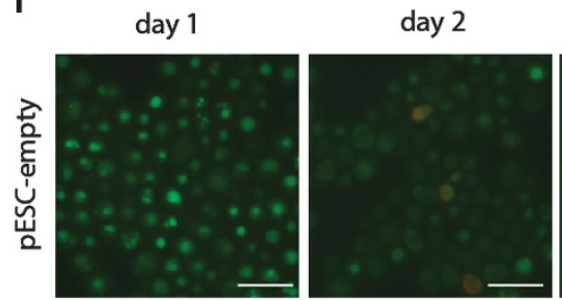

day 4
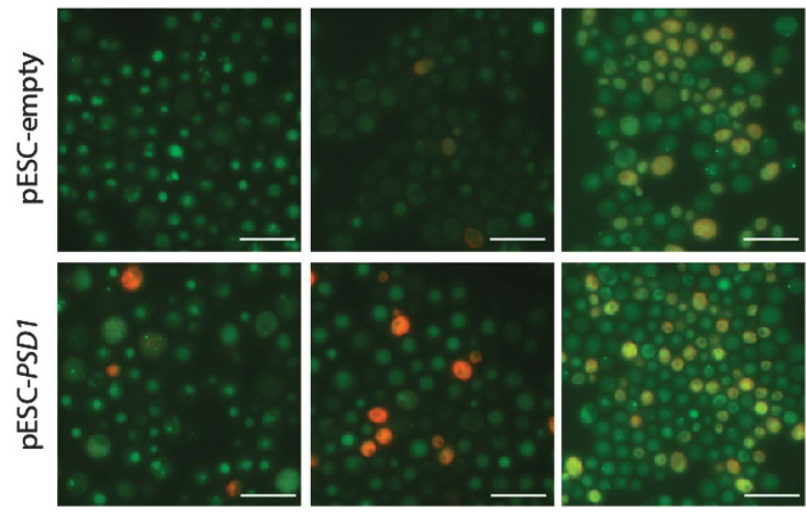

Figure 2 PSD1 overexpression extends chronological lifespan of wild-type yeast and increases autophagy. (a) Representative immunoblot monitoring Psd1 overexpression levels in conditions of chronological ageing. Full length Psd1-flag was detected by anti-Psd1 and anti-flag antibody at $\sim 55 \mathrm{kDa}$, whereas the cleaved protein (active form ${ }^{57}$ ) was only detected by the specific Psd1 antibody at $\sim 50 \mathrm{kDa}$. GAPDH abundance was assayed as loading control. (b) HPLC-ELSD-assisted quantification of total cellular phosphatidyl ethanolamine (PE) levels. (c) Clonogenic survival (d) ROS-driven conversion of DHE to ethidium in yeast chronological ageing is shown in control cells and in cells that overexpress PSD1. (e) Detection of GFP-Atg8 and GFP by immunoblotting at different days of ageing of cells that normally or overexpress PSD1. The band corresponding to free GFP (liberated from GFP-Atg8) is regarded as a measure of autophagy flux. (f) Representative GFP-Atg8 microscopy images of PSD1-overexpressing cells at day 1, 2 and 4 of chronological ageing. Scale bar $=10 \mu \mathrm{m}$

of autophagy stimulation. Autophagy induction by ethanolamine was neither associated with a decrease in mTORC1 activity, as measured by the phosphorylation of $p 70^{s 6 \mathrm{k}}$, nor with an activation of AMPK (Supplementary Figure S4D). All these experiments were repeated in a $\mathrm{H} 4$ neuroblastoma cell line stably expressing GFP-LCD, yielding similar results (data not shown). Hence, ethanolamine can stimulate autophagy in a variety of human cell lines. To assess cell survival, we finally performed a yeast-like chronological senescence assay, where highly confluent U2OS cells were left untreated or stimulated with $10 \mathrm{mM}$ ethanolamine for 1 week. Remarkably, ethanolamine treatment largely prevented the senescence-induced decrease of clonogenic cell survival (Figure 4d). In fact, ethanolamine was almost as effective as the potent autophagy stimulator rapamycin in improving human cell survival in over-confluent conditions. 


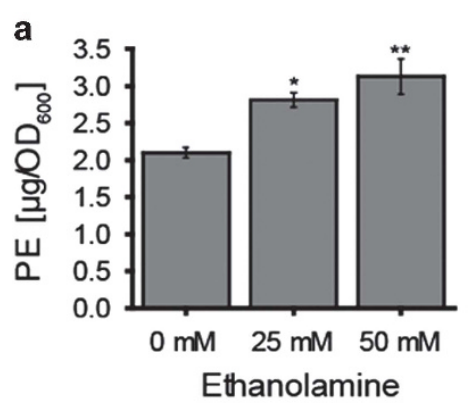

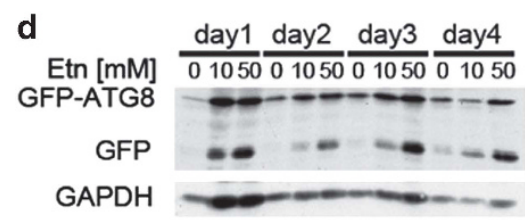

e
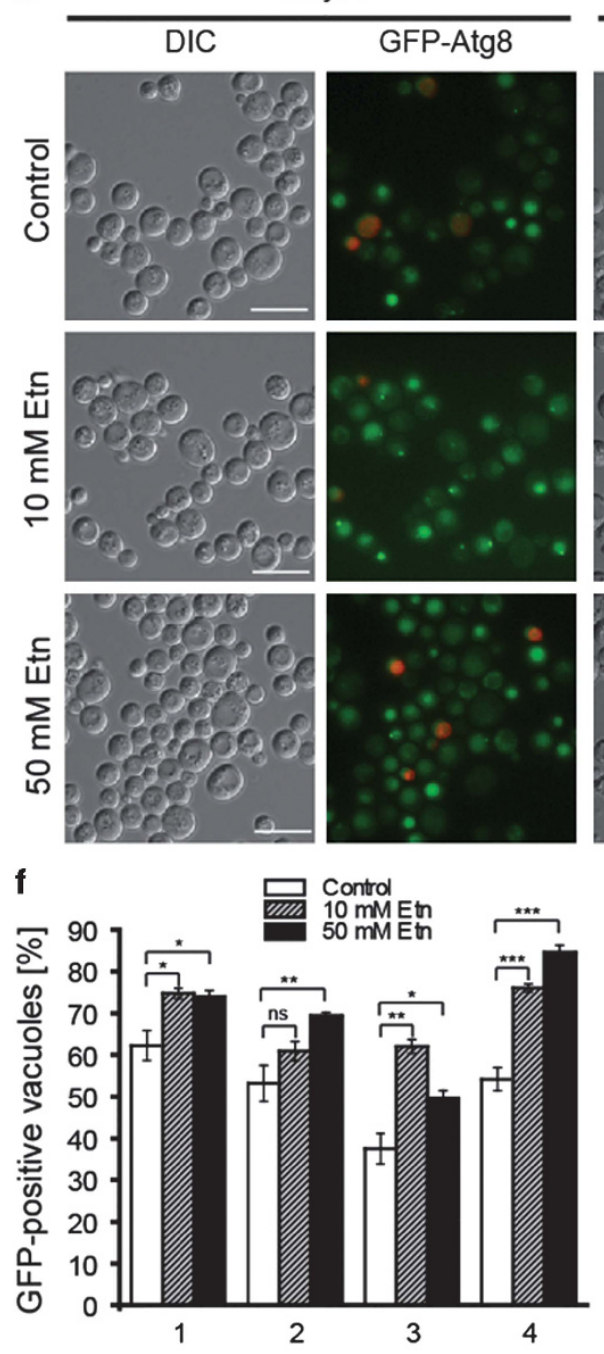

Day of ageing b

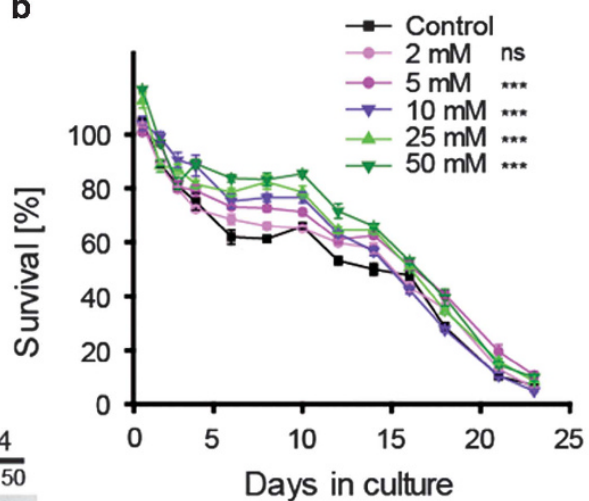

Day 3
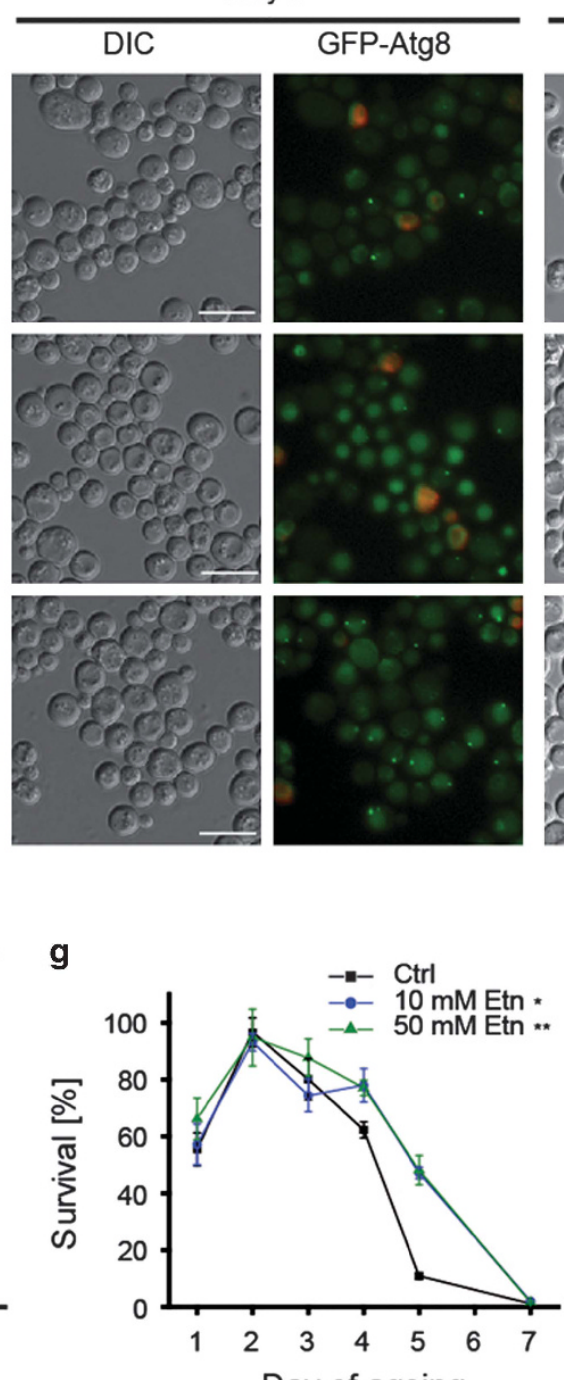

Day of ageing

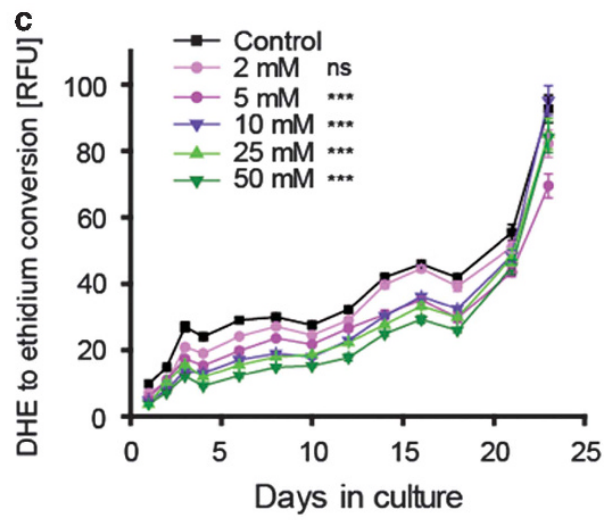

Day 4
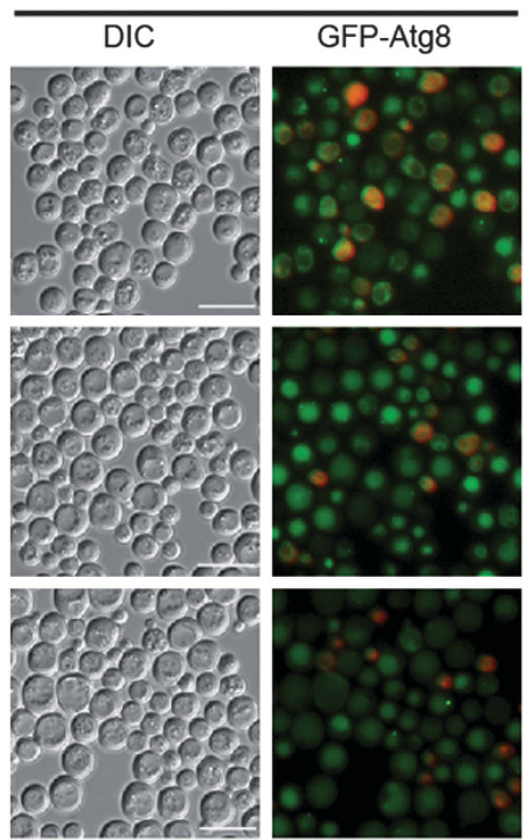

Figure 3 Administration of ethanolamine enhances the endogenous PE pool, induces autophagy and extends yeast lifespan. (a) HPLC-ELSD-assisted measurement of total cellular PE levels. (b) Clonogenic survival plating and (c) DHE to ethidium conversion throughout yeast chronological ageing upon addition of the indicated doses of ethanolamine. (d) Representative GFP-Atg8 immunoblot. The band corresponding to free GFP (liberated from GFP-Atg8) is considered as a measure of autophagic flux. (e) Representative microphotographs of GFP-Atg8-expressing cells under conditions of ethanolamine (Etn) administration. Scale bar $=5 \mu \mathrm{m}$. (f) GFP-Atg8 positive vacuoles were quantified in live (PI-negative) cells. (g) Chronological ageing of BY4742 wild type (left panel) and atg7 $\Delta$ (right panel) with 0,10 and 50 mM ethanolamine treatment 
a
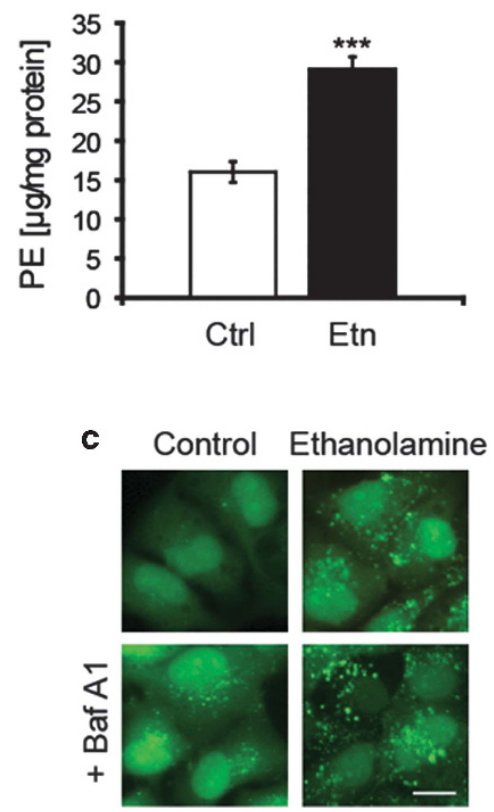

d

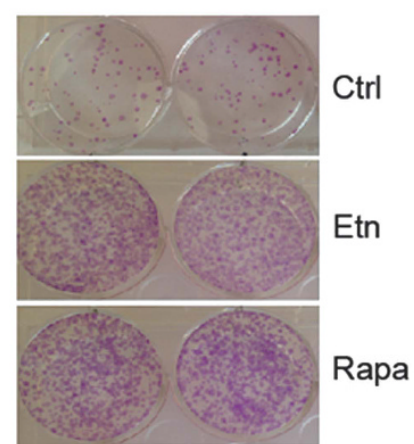

b
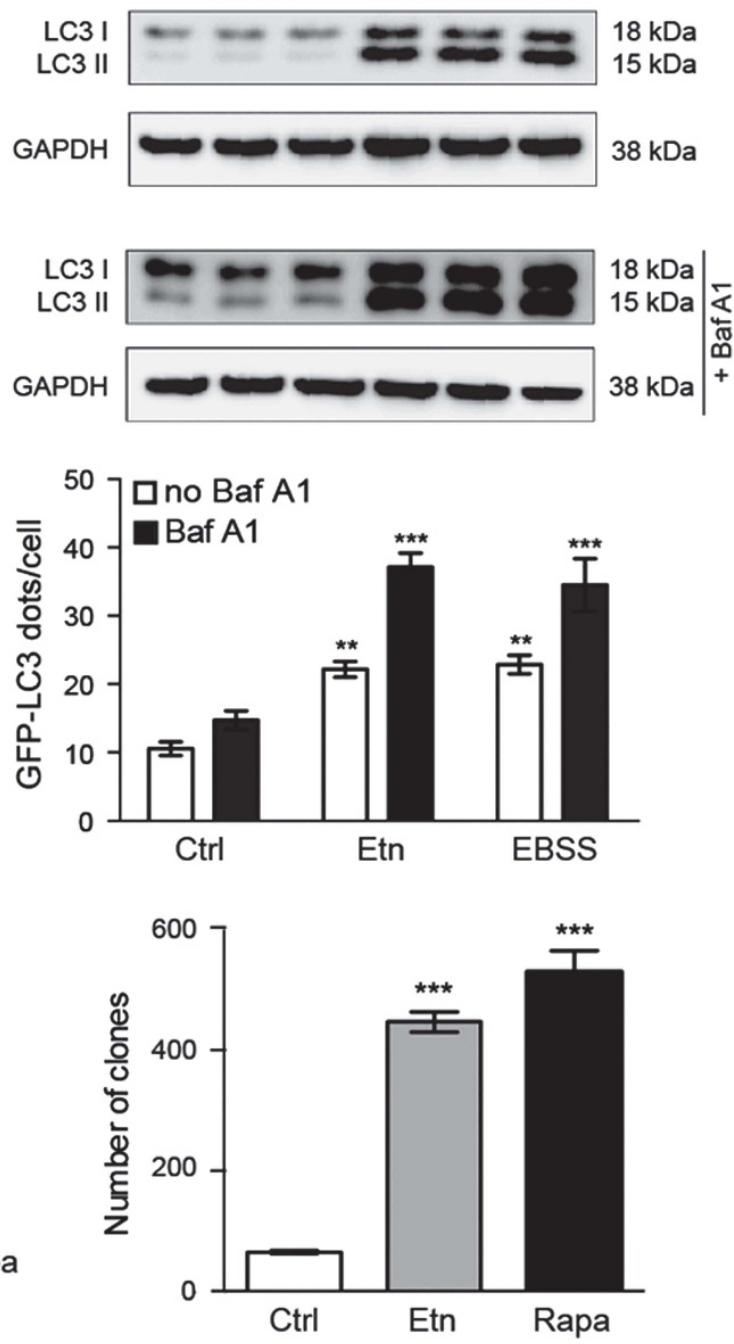

Figure 4 Administration of ethanolamine induces autophagy and reduces chronological senescence in mammalian cells. (a) HPLC-ELSD-assisted measurement of U2OS total cellular PE levels with and without $10 \mathrm{mM}$ ethanolamine (Etn) treatment. (b, c) Effect of ethanolamine administration on autophagy induction. Wild-type human osteosarcoma U2OS cells (b) and their GFP-LC3 expressing counterparts (c) were either left untreated or treated with $10 \mathrm{mM}$ ethanolamine. Thereafter, cells were processed for the immunochemical detection of LC3 lipidation (b) and for fluorescence microscopic quantification of GFP-LC3 positive dots (c). Administration of ethanolamine resulted in increased autophagy flux, as measured by LC3 lipidation in the absence or in the presence of bafilomycin A1 (bafA1). GAPDH levels were detected to ensure equal loading. Representative immunoblots are depicted in (b). Autophagy induction by ethanolamine treatment was confirmed by microscopic detection of GFP-LC3 ${ }^{+}$puncta in U2OS cells stably expressing GFP-LC3, in the absence or in the presence of bafilomycin A1 (c). Nutrient deprivation (EBSS) was used as a positive control of autophagy stimulation. (d) Ethanolamine reduces yeast-like chronological senescence and increases replicative viability in mammalian cells. Highly confluent U2OS cells were left untreated or stimulated with $10 \mathrm{mM}$ ethanolamine or $1 \mu \mathrm{M}$ rapamycin (Rapa, positive control) for one week

Ethanolamine increases lifespan in Drosophila. We finally evaluated the effect of ethanolamine on the fruit fly, Drosophila melanogaster. We supplied flies with ethanolamine and monitored their lifespan in five independent analyses as compared with untreated controls (Supplementary Figure S5). The pooled results revealed that the mean lifespan of flies treated with $10 \mathrm{mM}$ ethanolamine is significantly extended by $\sim 5 \%$ (Figure 5 ).

\section{Discussion}

Autophagy is regarded as one of the major cytoprotective mechanisms during ageing, ${ }^{12}$ and thus is a crucial process to counteract age-associated pathologies. Age-associated neurodegenerative disorders including Alzheimer's and Parkinson's disease may be postponed or attenuated by chronic induction of autophagy, ${ }^{12,39}$ and there is substantial evidence that genetic or pharmacological induction of autophagy can increase the healthspan and lifespan of multiple model organisms including yeast, worms, flies and mice. ${ }^{40}$ These findings have spurred the interest in identifying novel, nontoxic pharmacological inducers of autophagy. So far, several agents have been shown to induce autophagy and increase lifespan across several species, ${ }^{40}$ namely rapamycin, ${ }^{27,40}$ resveratrol ${ }^{40-42}$ and spermidine. ${ }^{43}$ The present results suggest that ethanolamine might be yet another potent autophagy inducer that promotes longevity. Like rapamycin, resveratrol and spermidine, ethanolamine is a naturally occurring 


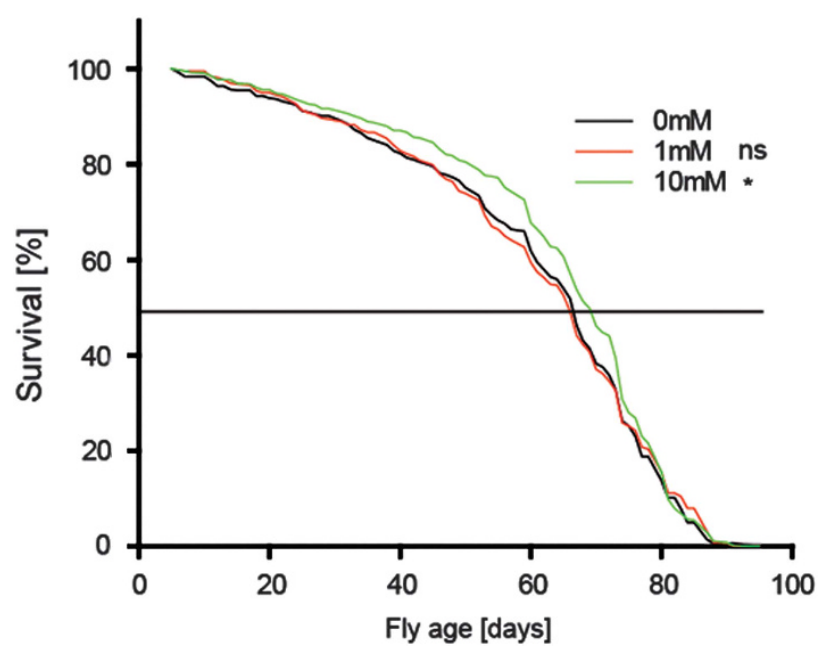

Figure 5 Ethanolamine administration enhances the lifespan of Drosophila melanogaster. Pooled data of five independent female fly lifespan measurements. The mean lifespan of the control population is $60.98 \pm 0.96,60.44 \pm 0.98$ for flies treated with $1 \mathrm{mM}$ ethanolamine and $64.05 \pm 0.90$ days for $10 \mathrm{mM}$ ethanolaminetreated flies. Wilcoxon comparison of $1 \mathrm{mM}$ treated flies with control flies revealed a $\chi^{2}$ of 0.2 on 1 degree of freedom and $P=0.688$, whereas a significant lifespan increase was assessed comparing $10 \mathrm{mM}$ with untreated flies with $\mathrm{a} \chi^{2}$ of 5.5 on 1 degree of freedom and $P=0.0192$. See Supplementary Figure S5 for individual lifespan analyses

component that is endowed with a favourable toxicity profile (note that the oral LD50 in rats is $1.72 \mathrm{~g} / \mathrm{kg}$ ) when compared with rapamycin and resveratrol.

Our study provides evidence that ethanolamine-mediated autophagy induction correlates with enhanced longevity in yeast and mammalian cell culture. This is in line with a previous study in yeast demonstrating that PE is a limiting factor for autophagy in a carboxypeptidase Y-defective background. ${ }^{44}$ Our results demonstrate that these observations are applicable to a wild-type scenario in yeast too and can be extended to mammalian cell cultures. Still, future experiments will need to clarify if ethanolamine-induced autophagy is beneficial to higher organisms. We could indeed observe a significant increase in the mean lifespan of flies upon supplementation with ethanolamine, but whether this is because of autophagy must be tackled in the follow-up studies.

Besides the pharmacological application of ethanolamine as an anti-ageing agent, our results underline the importance of this molecule for autophagy regulation. In vivo, phosphatidylserine decarboxylase plays a major role in the supply of $\mathrm{PE}$, which is a major component of mitochondrial membranes in yeast ${ }^{45}$ and mammals. ${ }^{1}$ It is thus not surprising that the abrogation of yeast $P S D 1$ or mammalian PISD affects mitochondrial dynamics such as mitochondrial fusion processes ${ }^{32,33,46}$ and reduces yeast lifespan (this study). Interestingly, in psd $1 \Delta \mathrm{Rho}^{0}$ the survival rates at day 1 exceed those of the $\mathrm{Rho}^{+}$counterpart, which suggests that a mitochondrial signal might be involved as a cell death trigger. Similarly, the phenotype of PISD homozygous knock-out mice is so severe that embryos die at day 8 to 10 of embryonic development. ${ }^{46}$ In another yeast study Psd2 has been described as being important for PE synthesis and autophagy under conditions of cadmium stress. ${ }^{47}$ This is interesting as it generally underlines the importance of PE-abundance for functional autophagy. However, in most of our experiments we observed stronger effects relating to Psd1 function. We showed that $P S D 1$ overexpression induced autophagy and increased the yeast lifespan. Genetic abrogation of autophagy-related factors in the overexpression setting would in addition strengthen the hypothesis that autophagy was crucial for lifespan extension, which will be tackled in a followup study. Our yeast and mammalian data indicate that ethanolamine treatment triggers autophagy through a phylogenetically conserved pathway. Moreover, at least in mammalian cells autophagy induction by ethanolamine was neither associated with a decrease in MTORC1 activity, as measured by the phosphorylation of $\mathrm{p} 70^{\mathrm{s} 6 \mathrm{k}}$, nor with an activation of AMPK (Supplementary Figure S4). Although we cannot exclude that alternative regulation processes are involved, it is possible that the availability of PE alone is sufficient to signal autophagy induction.

Of note, lipid determination during chronological ageing, might be diffused to some extent, as lipid extracts are not solely derived from living, but also from dead cells, which might still reside lipolytic activity and thus compromise the results of lipid profiling. However, this should not only affect PE content but similarly other phospholipid classes such as PC, PI or PS. Using the same lipid extracts as for PE-detection, PCmeasurement did not reveal significant change for $p s d 2 \Delta$, but slightly decreased PC levels in psd1 $\triangle$ compared with wild type (Figure 1c). Although PC is only reduced by $28 \%$ comparing psd1 to wild type on day 3 of chronological ageing, the PE reduction measures $77 \%$ (Figure 1c). Thus, we rather interpret the $\mathrm{PE}$ reduction in $p s d 1 \Delta$ and $p s d 2 \Delta$ as a predominant consequence of their primary loss of enzymatic function and not as a result of the dead cell population.

One suitable marker of autophagy is the PE-lipidation of LC3/Atg8, ${ }^{20}$ as we use it in this study. However, PE-lipidated LC3 has also been shown to localise to lipid droplets and to contribute to their formation. ${ }^{48,49}$ The LC3 conjugation system thus seems to be involved in lipid metabolism. In turn, autophagy has also been shown to be directly involved in lipid turnover. ${ }^{50}$ These studies show that lipid metabolism/storage and autophagy share some functional features. It is thus tempting to speculate that PE may act as a crucial molecular (and regulatory) link between autophagy and lipid metabolism.

Altogether, these results have identified ethanolamine as an autophagy-inducing stimulus that will be relevant for future autophagy, lipid and ageing research. Given its anti-ageing potential, ethanolamine might even emerge as a new therapeutic agent in the treatment of age-associated human diseases.

\section{Materials and Methods}

Yeast strains and growth conditions. All experiments were carried out in the BY4741 (MATa his $3 \Delta 1$ leu2 $\Delta 0$ met15 $\Delta 0$ ura3 $\Delta 0$ ) strain background except for the experiments shown in Figures $3 \mathrm{~g}$ and $\mathrm{h}$, where BY4742 (MAT $\alpha$ his3 11 leu2 $\Delta 0$ lys2 $\Delta 0$ ura3 $\Delta 0$ ) was used. Single deletion strains were obtained from the EUROSCARF knock-out collection (see Supplementary Table S1 for a complete list of strains). Strains were grown in SC medium containing $0.17 \%$ yeast nitrogen base (Difco, BD, Franklin Lakes, NJ, USA), $0.5 \%\left(\mathrm{NH}_{4}\right)_{2} \mathrm{SO}_{4}$ and $30 \mathrm{mg} / \mathrm{l}$ of all amino acids (except $80 \mathrm{mg} / \mathrm{l}$ histidine and $200 \mathrm{mg} / \mathrm{l}$ leucine), $30 \mathrm{mg} / \mathrm{l}$ adenine, and $320 \mathrm{mg} / \mathrm{l}$ uracil with $2 \%$ glucose as carbon source for SCD medium or $2 \%$ galactose for SCG 
medium, respectively. All yeast cultures were inoculated from a stationary overnight culture to an $\mathrm{OD}$ of 0.1 at $600 \mathrm{~nm}\left(\mathrm{OD}_{600}=0.1\right)$ and then grown at $28^{\circ} \mathrm{C}$ and $145 \mathrm{r}$. p.m. shaking for the indicated time periods. For induction of the Gal10 promoter to drive PSD1 expression, pESC-PSD1 containing cells were shifted to SCG medium at an $\mathrm{OD}_{600}=0.35$. Ethanolamine chloride from Sigma (St. Louis, MO, USA, E6133) was administered to the cultures from a $1 \mathrm{M}$ stock in $\mathrm{ddH}_{2} \mathrm{O}$ or from a $5 \mathrm{M}$ stock for the $50 \mathrm{mM}$ final concentration.

Cloning and molecular biology. PSD1 was cloned into the multiple cloning site 2 of the plasmid pESC His (Agilent Technologies, Santa Clara, CA, USA) by homologous recombination in yeast using the forward primer 5'-TTCGAATTCAACCCTCACTAAAGGGCGGCC_ATGTCAATTATGCCAGTTAAGAAC-3' and as backward primer 5'-GATCTTATCGTCGTCATCCTTGTAATCCAT_TTTAAATC ATTCTTTCCAATTATGCCTAATTTC-3'.

Survival plating and ROS determination. For survival plating, the cell concentrations of culture dilutions were determined with a CASY cell counter (Roche Diagnostics, Mannheim, Germany) and aliquots containing 500 cells were plated on YPD plates. The number of colonies formed was determined after 2 days at $28^{\circ} \mathrm{C}$. For dihydroethidium staining, $5 \times 10^{6}$ cells were harvested by centrifugation, resuspended in $250 \mu \mathrm{l}$ of $2.5 \mu \mathrm{g} / \mathrm{ml}$ DHE in PBS and incubated in the dark for $5 \mathrm{~min}$. Relative fluorescence units were determined using a Tecan GeniusPRO fluorescence reader (Tecan Group, Maennedorf, Switzerland) and then normalised to $\mathrm{OD}_{600}$. For ROS analysis on the basis of individual cells, flow cytometry was used to count the positive cells.

Yeast autophagy measurement. Autophagy was measured by monitoring the cytosol to vacuole translocation of Atg8 using fluorescence microscopy or immunoblotting (GFP liberation assay) of cells/cell extracts from strains carrying a GFP-Atg8 fusion protein ${ }^{34,35}$ expressed under its endogenous promoter and at its natural chromosomal locus. Quantification of micrographs was performed from blinded pictures with 150-300 cells per micrograph and replicate. Autophagic cells were defined as cells exhibiting clear vacuolar GFP fluorescence and expressed as fraction of viable (PI-negative) cells. Immunoblotting followed standard procedures using anti-GFP (Roche Diagnostics, \#11814460001), anti-Psd1 or antiglyceraldehyde-3-phosphate dehydrogenase (GAPDH) antibodies (both gifts from Dr. Günther Daum).

Yeast lipid extraction. Total lipids were extracted from exponentially growing yeast cultures at indicated days of ageing with chloroform/methanol 2:1 (v/v) according to Folch et al. ${ }^{51}$ The organic phase was dried under a stream of nitrogen and dissolved in $500 \mu \mathrm{l}$ of chloroform/methanol $(2: 1, \mathrm{v} / \mathrm{v})$.

Lipid analysis. PE was quantified by a normal phase HPLC_-evaporative light scattering detector (ELSD) method as described in Guerfal et al. ${ }^{52}$ (the method is accepted for publication in Cold Spring Harbor Protocols: 'Analyzing and understanding lipids of yeast: a challenging endeavour'). In brief, the chromatographic separation of lipids was achieved on a Betasil Diol column $(100 \times 4.6 \mathrm{~mm}$, particle size $5 \mu \mathrm{m}$, Thermo Fisher Scientific Inc., Waltham, MA, USA) with a ternary gradient (modified from 53 and described in detail in Cold Spring Harbor Protocols). PE 34:1 standard (Avanti Polar Lipids, Inc., Alabaster, AL, USA) was prepared as $1 \mathrm{mg} / \mathrm{ml}$ stock solution in chloroform/methanol 2:1 (v/v). Calibration curves (triplicates) were measured from 2.7 to $350 \mu \mathrm{g} / \mathrm{ml}, 10 \mu \mathrm{l}$ sample was injected for each measurement.

\section{Cell culture}

Chemicals, cell lines and culture conditions: Unless otherwise specified, chemicals were purchased from Sigma-Aldrich (St. Louis, MO, USA), culture media and supplements for cell culture from Gibco-Invitrogen (Carlsbad, CA, USA) and plasticware from Corning (Corning, NY, USA). Human osteosarcoma U2OS cells, their GFP-LC3-expressing derivatives, human neuroblastoma H4 GFP-LC3 (gift from $Y$. Juan) cells were cultured in DMEM medium containing $10 \%$ foetal bovine serum, $100 \mathrm{mg} / \mathrm{l}$ sodium pyruvate, $10 \mathrm{mM}$ HEPES buffer, $100 \mathrm{units} / \mathrm{ml}$ penicillin $\mathrm{G}$ sodium and $100 \mu \mathrm{g} / \mathrm{ml}$ streptomycin sulphate $\left(37^{\circ} \mathrm{C}, 5 \% \mathrm{CO}_{2}\right)$. Lipid extractions were performed after $12 \mathrm{~h}$ of $10 \mathrm{mM}$ ethanolamine (Sigma, E9508) treatment. For autophagy induction, cells were treated for $12 \mathrm{~h}$ with $10 \mathrm{mM}$ ethanolamine or incubated in absence of nutrients. For the yeast-like chronological senescence assay, cells were treated for 1 week with $10 \mathrm{mM}$ ethanolamine or $1 \mu \mathrm{M}$ rapamycin (R\&D Systems, Minneapolis, MN, USA).
Lipid extraction from U2OS and H4 cells: Cells were harvested in PBS pH 7.4 and pelleted before shock freezing in liquid nitrogen. Cell disruption was performed by sonication in PBS $\mathrm{pH}$ 7.4. Protein concentration was determined using the Bio-Rad protein assay kit (Bio-Rad Laboratories, Hercules, CA, USA) and the results were used for normalisation after HPLC measurement. The raw extracts were extracted with chloroform/methanol (2:1) after Folch. ${ }^{51}$ The organic phase was collected and combined with the organic phase obtained from the re-extraction of the aqueous phase. Organic solvents were evaporated under a stream of nitrogen and the lipids were dissolved in chloroform/ methanol (2:1). Lipid analysis was performed as described above.

Immunoblotting. For immunoblotting, $25 \mu \mathrm{g}$ of proteins were separated on $4-12 \%$ bis-tris acrylamide (Thermo Fisher Scientific Inc.) and electrotransferred to Immobilon membranes (Merck Millipore, Darmstadt, Germany). Membranes were then sliced horizontally in different parts according to the molecular weight of the protein of interest to allow simultaneous detection of different antigens within the same experiment. ${ }^{54,55}$ Unspecific binding sites were saturated by incubating membranes for $1 \mathrm{~h}$ in $0.05 \%$ Tween 20 (v:v in TBS) supplemented with 5\% non-fat powdered milk (w:v in TBS), followed by an overnight incubation with primary antibodies specific for LC3B, phospho-AMPK (Thr172), AMPK, phospho-ribosomal protein S6 kinase (Thr421/ Ser424), ribosomal protein S6 kinase (Cell Signalling Technology Inc., Danvers, MA, USA). Development was performed with appropriate horseradish peroxidase (HRP)labelled secondary antibodies (Southern Biotech, Birmingham, AL, USA) plus the SuperSignal West Pico chemoluminescent substrate (Thermo Fisher Scientific Inc.). An anti-glyceraldehyde-3-phosphate dehydrogenase antibody (Chemicon International Inc., Temecula, CA, USA) was used to control equal loading of lanes.

Automated microscopy. U2OS or $\mathrm{H} 4$ cells stably expressing GFP-LC3 were seeded in 96-well imaging plates (BD Falcon, Sparks, USA) $24 \mathrm{~h}$ before stimulation. Cells were treated with the indicated agents for $4 \mathrm{~h}$. Subsequently, cells were fixed with 4\% PFA and counterstained with $10 \mu \mathrm{M}$ Hoechst 33342. Images were acquired using a BD pathway 855 automated microscope (BD Imaging Systems, San José, USA) equipped with a 40X objective (Olympus, Center Valley, USA) coupled to a robotised Twister II plate handler (Caliper Life Sciences, Hopkinton, USA). Images were analyzed for the presence of GFP-LC3 puncta in the cytoplasm by means of the BD Attovision software (BD Imaging Systems). Cell surfaces were segmented and divided into cytoplasmic and nuclear regions according to manufacturer standard proceedings. RB 2x2 and Marr-Hildreth algorithms were used to recognize cytoplasmic GFP-LC3 positive dots.

Yeast-like chronological senescence assay. The assay was performed as described in Leontieva et al. ${ }^{56}$ Briefly, 80000 cells were seeded into 96-well plates and left untreated or treated with $10 \mathrm{mM}$ ethanolamine or $1 \mu \mathrm{M}$ rapamycin. After 8 days, dead cells and conditioning media were removed, cells were trypsinized and a 10\% aliquot was plated in fresh medium-filled six-well plates. After 1 week, clones were marked trough crystal violet staining and counted.

Drosophila lifespan experiments. Female flies from an isogenised $w^{1118}$ strain were used. They were kept in a $25^{\circ} \mathrm{C}, 70 \%$ humidity, $12 \mathrm{~h}$ light/12 $\mathrm{h}$ dark incubator on a standard cornmeal-sugar-yeast diet. Flies were collected at emergence and 20 females were kept per vial with an average of 87 flies per group and replicate. The food was changed twice a week and supplemented or not with ethanolamine hydrochloride (Sigma, E6133) at $1 \mathrm{mM}$ or $10 \mathrm{mM}$ final concentrations. The number of dead flies were recorded every weekday until all flies were dead. Five independent replicates were performed. The independent replicates, as well as the pooled data from all replicates were analysed by a Wilcoxon survival analysis test in $\mathrm{R}$ (script available on request).

Statistical analysis. Statistical analyses were calculated in Origin8. For assessment of significance one-way ANOVA followed by Bonferroni post hoc test was performed, except for Figure 1b, Figures $2 a$ and $b$ and Figures $3 b, c, g$ and $h$ which were processed using a two-factor ANOVA with strain and time as independent factors. Data in Figure 5 were assessed for significant difference by Wilcoxon analysis. Error bars indicate standard error of the mean (SEM) and asterisks in the figures indicate significant differences, ${ }^{*} P<0.05,{ }^{\star \star} P<0.01,{ }^{* \star \star} P<0.001$.

\section{Conflict of Interest}

The authors declare no conflict of interest. 
Acknowledgements. This work was supported by the Austrian Science Fund FWF (grants LIPOTOX, I1000-B20, P23490-B12, and P24381-B20) to FM. We acknowledge support from NAWI Graz. OK is a member of the PhD program 'Molecular Enzymology', funded by the FWF (project W901-B12). GK is supported by the Ligue contre le Cancer (équipe labelisée); Agence National de la Recherche (ANR); Association pour la recherche sur le cancer (ARC); Cancéropôle lle-deFrance; Institut National du Cancer (INCa); Fondation Bettencourt-Schueller; Fondation de France; Fondation pour la Recherche Médicale (FRM); the European Commission (ArtForce); the European Research Council (ERC); the LabEx ImmunoOncology; the SIRIC Stratified Oncology Cell DNA Repair and Tumour Immune Elimination (SOCRATE); the SIRIC Cancer Research and Personalized Medicine (CARPEM); and the Paris Alliance of Cancer Research Institutes (PACRI). We thank Günther Daum for the kind gift of the specific Psd1 antibody.

1. Vance JE. Phosphatidylserine and phosphatidylethanolamine in mammalian cells: two metabolically related aminophospholipids. J Lipid Res 2008; 49: 1377-1387.

2. Schuiki I, Schnabl M, Czabany T, Hrastnik C, Daum G. Phosphatidylethanolamine synthesized by four different pathways is supplied to the plasma membrane of the yeast Saccharomyces cerevisiae. Biochim Biophys Acta 2010; 1801: 480-486.

3. Clancey CJ, Chang SC, Dowhan W. Cloning of a gene (PSD1) encoding phosphatidylserine decarboxylase from Saccharomyces cerevisiae by complementation of an Escherichia coli mutant. J Biol Chem 1993; 268: 24580-24590.

4. Zinser E, Sperka-Gottlieb CD, Fasch EV, Kohlwein SD, Paltauf F, Daum G. Phospholipid synthesis and lipid composition of subcellular membranes in the unicellular eukaryote Saccharomyces cerevisiae. J Bacteriol 1991; 173: 2026-2034.

5. Trotter PJ, Voelker DR. Identification of a non-mitochondrial phosphatidylserine decarboxylase activity (PSD2) in the yeast Saccharomyces cerevisiae. J Biol Chem 1995; 270: $6062-6070$

6. Nikawa J, Tsukagoshi Y, Yamashita S. Cloning of a gene encoding choline transport in Saccharomyces cerevisiae. J Bacteriol 1986; 166: 328-330.

7. Kim K, Kim KH, Storey MK, Voelker DR, Carman GM. Isolation and characterization of the Saccharomyces cerevisiae EKI1 gene encoding ethanolamine kinase. J Biol Chem 1999; 274: $14857-14866$

8. Min-Seok R, Kawamata Y, Nakamura H, Ohta A, Takagi M. Isolation and characterization of ECT1 gene encoding CTP: phosphoethanolamine cytidylyltransferase of Saccharomyces cerevisiae. J Biochem 1996; 120: 1040-1047.

9. Hjelmstad RH, Bell RM. The sn-1,2-diacylglycerol ethanolaminephosphotransferase activity of Saccharomyces cerevisiae. Isolation of mutants and cloning of the EPT1 gene. J Biol Chem 1988; 263: 19748-19757.

10. van den Brink-van der Laan E, Killian JA, de Kruijff B. Nonbilayer lipids affect peripheral and integral membrane proteins via changes in the lateral pressure profile. Biochim Biophys Acta 2004; 1666: 275-288

11. Nakatogawa $H$, Ichimura $Y$, Ohsumi $Y$. Atg8, a ubiquitin-like protein required for autophagosome formation, mediates membrane tethering and hemifusion. Cell 2007; 130 : 165-178.

12. Feng Y, He D, Yao Z, Klionsky DJ. The machinery of macroautophagy. Cell Res 2014; 24 24-41.

13. Inoue Y, Klionsky DJ. Regulation of macroautophagy in Saccharomyces cerevisiae. Semin Cell Dev Biol 2010; 21: 664-670.

14. Luo $S$, Chen $Q$, Cebollero $E$, Xing D. Mitochondria: one of the origins for autophagosoma membranes? Mitochondrion 2009; 9: 227-231.

15. Yang Z, Klionsky DJ. Eaten alive: a history of macroautophagy. Nat Cell Biol 2010; 12 814-822.

16. Hailey DW, Rambold AS, Satpute-Krishnan P, Mitra K, Sougrat R, Kim PK et al. Mitochondria supply membranes for autophagosome biogenesis during starvation. Cell 2010; 141 : 656-667.

17. Reunanen $\mathrm{H}$, Punnonen EL, Hirsimaki P. Studies on vinblastine-induced autophagocytosis in mouse liver. V. A cytochemical study on the origin of membranes. Histochemistry 1985; 83 513-517.

18. Bleijerveld $\mathrm{OB}$, Brouwers JF, Vaandrager AB, Helms JB, Houweling M. The CDP. ethanolamine pathway and phosphatidylserine decarboxylation generate different phosphatidylethanolamine molecular species. J Biol Chem 2007; 282: 28362-28372.

19. Reggiori F, Shintani T, Nair U, Klionsky DJ. Atg9 cycles between mitochondria and the preautophagosomal structure in yeasts. Autophagy 2005; 1: 101-109.

20. Ichimura Y, Kirisako T, Takao T, Satomi Y, Shimonishi Y, Ishihara N et al. A ubiquitin-like system mediates protein lipidation. Nature 2000; 408: 488-492.

21. Kabeya Y, Mizushima N, Ueno T, Yamamoto A, Kirisako T, Noda T et al. LC3, a mammalian homologue of yeast Apg8p, is localized in autophagosome membranes after processing EMBO J 2000; 19: 5720-5728

22. Tanida I, Sou YS, Ezaki J, Minematsu-lkeguchi N, Ueno T, Kominami E. HsAtg4B/ HsApg4B/autophagin-1 cleaves the carboxyl termini of three human Atg8 homologues and delipidates microtubule-associated protein light chain 3- and GABAA receptor-associated protein-phospholipid conjugates. J Biol Chem 2004; 279 36268-36276.
23. Fujita $\mathrm{N}$, Hayashi-Nishino M, Fukumoto $\mathrm{H}$, Omori $\mathrm{H}$, Yamamoto $\mathrm{A}$, Noda T et al. An Atg4B mutant hampers the lipidation of LC3 paralogues and causes defects in autophagosome closure. Mol Biol Cell 2008; 19: 4651-4659.

24. Kirisako T, Ichimura Y, Okada H, Kabeya Y, Mizushima N, Yoshimori T et al. The reversible modification regulates the membrane-binding state of Apg8/Aut7 essential for autophagy and the cytoplasm to vacuole targeting pathway. J Cell Biol 2000; 151: 263-276.

25. Fujita N, Itoh T, Omori H, Fukuda M, Noda T, Yoshimori T. The Atg16 complex specifies the site of LC3 lipidation for membrane biogenesis in autophagy. Mol Biol Cell 2008; 19: 2092-2100.

26. Hanada T, Noda NN, Satomi Y, Ichimura Y, Fujioka Y, Takao T et al. The Atg12-Atg5 conjugate has a novel E3-like activity for protein lipidation in autophagy. J Biol Chem 2007; 282: 37298-37302.

27. Noda T, Ohsumi Y. Tor, a phosphatidylinositol kinase homologue, controls autophagy in yeast. J Biol Chem 1998; 273: 3963-3966.

28. Xie Z, Nair U, Klionsky DJ. Atg8 controls phagophore expansion during autophagosome formation. Mol Biol Cell 2008; 19: 3290-3298.

29. Yang A, Li Y, Pantoom S, Triola G, Wu YW. Semisynthetic lipidated LC3 protein mediates membrane fusion. Chembiochem 2013; 14: 1296-1300.

30. Almeida T, Marques M, Mojzita D, Amorim MA, Silva RD, Almeida B et al. Isc1p plays a key role in hydrogen peroxide resistance and chronological lifespan through modulation of iron levels and apoptosis. Mol Biol Cell 2008; 19: 865-876.

31. Burgermeister M, Birner-Grunberger R, Nebauer R, Daum G. Contribution of different pathways to the supply of phosphatidylethanolamine and phosphatidylcholine to mitochondrial membranes of the yeast Saccharomyces cerevisiae. Biochim Biophys Acta 2004; 1686: 161-168.

32. Birner R, Burgermeister M, Schneiter R, Daum G. Roles of phosphatidylethanolamine and of its several biosynthetic pathways in Saccharomyces cerevisiae. Mol Biol Cell 2001; 12 : 997-1007.

33. Chan EY, McQuibban GA. Phosphatidylserine decarboxylase 1 (Psd1) promotes mitochondrial fusion by regulating the biophysical properties of the mitochondrial membrane and alternative topogenesis of mitochondrial genome maintenance protein 1 (Mgm1). J Biol Chem 2012; 287: 40131-40139.

34. Kirisako T, Baba M, Ishihara N, Miyazawa K, Ohsumi M, Yoshimori T et al. Formation process of autophagosome is traced with Apg8/Aut7p in yeast. $J$ Cell Biol 1999; 147: 435-446.

35. Klionsky DJ, Cuervo AM, Seglen PO. Methods for monitoring autophagy from yeast to human. Autophagy 2007; 3: 181-206.

36. Noda T, Klionsky DJ. The quantitative Pho8Delta60 assay of nonspecific autophagy. Methods Enzymol 2008; 451: 33-42.

37. Noda T, Matsuura A, Wada Y, Ohsumi Y. Novel system for monitoring autophagy in the yeast Saccharomyces cerevisiae. Biochem Biophys Res Commun 1995; 210: 126-132.

38. Burtner CR, Murakami CJ, Kennedy BK, Kaeberlein M. A molecular mechanism of chronological aging in yeast. Cell Cycle 2009; 8: 1256-1270.

39. Rubinsztein DC, DiFiglia M, Heintz N, Nixon RA, Qin ZH, Ravikumar B et al. Autophagy and its possible roles in nervous system diseases, damage and repair. Autophagy 2005; 1: 11-22.

40. Madeo F, Tavernarakis N, Kroemer G. Can autophagy promote longevity? Nat Cell Biol 2010; 12: 842-846.

41. Howitz KT, Bitterman KJ, Cohen HY, Lamming DW, Lavu S, Wood JG et al. Smal molecule activators of sirtuins extend Saccharomyces cerevisiae lifespan. Nature 2003; 425: 191-196.

42. Wood JG, Rogina B, Lavu S, Howitz K, Helfand SL, Tatar M et al. Sirtuin activators mimic caloric restriction and delay ageing in metazoans. Nature 2004; 430: 686-689.

43. Eisenberg T, Knauer H, Schauer A, Buttner S, Ruckenstuhl C, Carmona-Gutierrez D et al. Induction of autophagy by spermidine promotes longevity. Nat Cell Biol 2009; 11: 1305-1314.

44. Nebauer R, Rosenberger S, Daum G. Phosphatidylethanolamine, a limiting factor of autophagy in yeast strains bearing a defect in the carboxypeptidase $Y$ pathway of vacuolar targeting. J Biol Chem 2007; 282: 16736-16743.

45. Tuller G, Nemec T, Hrastnik C, Daum G. Lipid composition of subcellular membranes of an FY1679-derived haploid yeast wild-type strain grown on different carbon sources. Yeast 1999; 15: 1555-1564.

46. Steenbergen R, Nanowski TS, Beigneux A, Kulinski A, Young SG, Vance JE. Disruption of the phosphatidylserine decarboxylase gene in mice causes embryonic lethality and mitochondrial defects. J Biol Chem 2005; 280: 40032-40040.

47. Muthukumar K, Nachiappan V. Phosphatidylethanolamine from phosphatidylserine decarboxylase2 is essential for autophagy under cadmium stress in Saccharomyces cerevisiae. Cell Biochem Biophys 2013; 67: 1353-1363.

48. Shibata M, Yoshimura K, Furuya N, Koike M, Ueno T, Komatsu M et al. The MAP1-LC3 conjugation system is involved in lipid droplet formation. Biochem Biophys Res Commun 2009; 382: 419-423.

49. Shibata M, Yoshimura K, Tamura H, Ueno T, Nishimura T, Inoue T et al. LC3, a microtubuleassociated protein1A/B light chain3, is involved in cytoplasmic lipid droplet formation. Biochem Biophys Res Commun 2010; 393: 274-279.

50. Singh R, Kaushik S, Wang Y, Xiang Y, Novak I, Komatsu M et al. Autophagy regulates lipid metabolism. Nature 2009; 458: 1131-1135.

51. Folch J, Lees M, Sloane Stanley GH. A simple method for the isolation and purification of total lipides from animal tissues. J Biol Chem 1957; 226: 497-509. 
52. Guerfal M, Claes K, Knittelfelder O, De Rycke R, Kohlwein SD, Callewaert N. Enhanced membrane protein expression by engineering increased intracellular membrane production. Microb Cell Fact 2013; 12: 122.

53. Graeve M, Janssen D. Improved separation and quantification of neutral and polar lipid classes by HPLC-ELSD using a monolithic silica phase: application to exceptional marine lipids. J Chromatogr B Analyt Technol Biomed Life Sci 2009; 877 1815-1819.

54. Criollo A, Niso-Santano M, Malik SA, Michaud M, Morselli E, Marino G et al. Inhibition of autophagy by TAB2 and TAB3. EMBO J 2011; 30: 4908-4920.

55. Shen S, Niso-Santano M, Adjemian S, Takehara T, Malik SA, Minoux H et al. Cytoplasmic STAT3 represses autophagy by inhibiting PKR activity. Mol Cell 2012; 48: 667-680.

56. Leontieva OV, Blagosklonny MV. Yeast-like chronological senescence in mammalian cells: phenomenon, mechanism and pharmacological suppression. Aging 2011; 3: 1078-1091.
57. Horvath SE, Bottinger L, Vogtle FN, Wiedemann N, Meisinger C, Becker T et al. Processing and topology of the yeast mitochondrial phosphatidylserine decarboxylase 1. J Biol Chem 2012; 287: 36744-36755.

(c) (1) This work is licensed under a Creative Commons Attribution 3.0 Unported License. The images or other third party material in this article are included in the article's Creative Commons license, unless indicated otherwise in the credit line; if the material is not included under the Creative Commons license, users will need to obtain permission from the license holder to reproduce the material. To view a copy of this license, visit http://creativecommons.org/ licenses/by/3.0/

Supplementary Information accompanies this paper on Cell Death and Differentiation website (http://www.nature.com/cdd) 